Automatized and desktop AC-susceptometer for the in situ and real time monitoring of magnetic nanoparticles' synthesis by coprecipitation

M. P. Fernández-García, J. M. Teixeira, P. Machado, M. R. F. F. Oliveira, J. M. Maia, C. Pereira, A. M. Pereira, C. Freire, and J. P. Araujo

This article may be downloaded for personal use only. Any other use requires prior permission of the author and AIP Publishing.

The following article appeared in Review of Scientific Instruments 86, 043904 (2015) and may be found at https://doi.org/10.1063/1.4918723. 


\title{
Automatized and desktop AC-susceptometer for the in situ and real time monitoring of magnetic nanoparticles synthesis by coprecipitation
}

\author{
M.P. Fernández-García ${ }^{1 a)}$, J.M. Teixeira1 ${ }^{1}$ P. Machado ${ }^{1}$, M.R.F.F. Oliveira², J.M. \\ Maia $^{1}$, C. Pereira ${ }^{3}$, A.M. Pereira ${ }^{1}$, C. Freire $^{3}$, J.P. Araujo ${ }^{1}$ \\ ${ }^{I}$ IFIMUP and IN-Institute of Nanoscience and Nanotechnology, Departamento de Física e Astronomía, Faculdade de \\ Ciências, Universidade do Porto, Portugal \\ ${ }^{2}$ Departamento de Ciência de Computadores, Faculdade de Ciências, Universidade do Porto, Portugal \\ ${ }^{3}$ REQUIMTE/LAQV, Departamento de Química e Bioquímica, Faculdade de Ciências, Universidade do Porto, Portugal
}

\begin{abstract}
The main purpose of this work was to design, develop and construct a simple desktop AC susceptometer to monitor in situ and in real time the coprecipitation synthesis of magnetic nanoparticles. The design incorporates one pair of identical pick-up sensing coils and one pair of Helmholtz coils. The picked up signal is detected by a lock-in SR850 amplifier that measures the in- and out-of-phase signals. The apparatus also includes a stirrer with $45^{\circ}$-angle blades to promote the fast homogenization of the reaction mixture. Our susceptometer has been successfully used to monitor the coprecipitation reaction for the synthesis of iron oxide nanoparticles.
\end{abstract}

\section{INTRODUCTION}

The synthesis, physicochemical characterization and applications of inorganic nanoparticles (NPs) have been explored for a long time. ${ }^{1-14}$ However, the interest on magnetic NPs (especially on iron oxides and transition metal nanoferrites) "strikes back" due to their broad perspectives and renewable applications ${ }^{15-22}$ but complicates their requirements and their desired properties. On the other hand, there is a gap between laboratory research and the scale-up for industrial production due to the difficulty to produce kilogram quantities of nanomaterial with the desired properties (commonly with dimensions smaller than $20 \mathrm{~nm}$, narrow particle size distribution and superparamagnetic behavior). Therefore, it is of major importance investing on synthesis processes that can be easily controllable, cost-effective and that lead to the large-scale production of magnetic nanomaterials.

One of the most common and easiest ways to synthesize magnetic iron oxides and ferrite NPs is by coprecipitation of ferrous and ferric salt solutions with a base (typically ammonia, alkyl tetraalkylammonium hydroxide and $\mathrm{NaOH}$ ) in an aqueous medium. ${ }^{12,23}$ This method is widely employed due to its simplicity, reduced costs, high NPs yields, and benignity for environment since it does not employ neither hazardous solvents nor high temperature / pressure conditions. ${ }^{24}$ Despite these virtues, the NPs produced by coprecipitation typically exhibit broad size distributions leading to costly and timeconsuming downstream purification and isolation processes. ${ }^{8}$ To overcome these drawbacks, a novel and

a) Author to whom correspondence should be addressed. Electronic mail: Maria Paz Fernández García (PhD): maria.garcia@fc.up.pt single-step route to induce the formation of Fe-oxide NPs by coprecipitation using alkanolamines as bases has been recently reported. ${ }^{23,25}$ These nanomaterials exhibited enhanced magnetic properties, when compared with ferrites of the same dimensions but obtained with other bases or by other synthesis routes. Simultaneously, the alkanolamines acted as complexing agents that controlled the particle size during the synthesis process.

The fabrication of magnetic NPs with detailed composition, crystal structure and magnetic properties requires a precisely controlled reproducibility of the synthesis procedures. The aqueous coprecipitation for the fabrication of iron oxide NPs is a kinetically-driven reaction so, small fluctuations of the reaction parameters (type of $\mathrm{Fe}$ salts; $\mathrm{Fe}(\mathrm{II}) / \mathrm{Fe}$ (III) molar ratio; reaction temperature; $\mathrm{pH}$ value; mixing velocity, etc.) are likely to influence the outcome of the reaction. ${ }^{16,26}$ Additionally, not only coprecipitation but all batch reactions have certain limitations when considered for industrial production. Consequently, the scale-up of the magnetic NPs synthesis processes could also be a problem due to the low production of small laboratory units, ${ }^{27}$ the inhomogeneous mixing and, the side reactions that may change from batchto-batch. ${ }^{26,28,29}$ The in situ and real time monitoring of the synthesis process constitutes a potential strategy for the complete understanding of the reaction mechanism involved in the NPs synthesis and of the influence of all reaction parameters. ${ }^{30}$ For that purpose, we have integrated an AC susceptometer within the mixing zone of the reagents to monitor in situ and in real time the NPs' growth and the evolution of their magnetic properties during the aqueous coprecipitation reaction. V. Ström and co-authors have previously studied the influence of chemical base concentration during the course of 
coprecipitation reactions with an AC susceptometer. ${ }^{30}$ They developed an external mixer to blend two jets (metal salt precursors and base) and afterwards, transferred the reaction mixture to a sample vial that was placed inside the AC susceptometer. On the contrary, our set-up monitors the coprecipitation reaction from the exact moment that the base is vigorously blended until the end of the synthesis. This allows us to follow the reaction from the first $\mu s$ only limited by the LABVIEW data acquisition program. For that purpose, we had to design and implement a hydrofoil impeller with $45^{\circ}$-angle blades to promote a fast homogenization of the reaction mixture.

Herein we report on a simple desktop AC susceptometer to monitor simultaneously the evolution of the in-phase and out-of-phase susceptibility of Fe-oxide NPs during the full course of coprecipitation reactions. Therefore, we can obtain in situ information of the reaction times and optimize them through the appropriate choice of the synthetic conditions. We will exemplify the potentialities of the developed setup to distinguish between diamagnetic, ferromagnetic and superparamagnetic materials and also show its resolution to distinguish between ferrite NPs with minute and null coercive field $\left(H_{c}\right)$ values. Finally, we will demonstrate its performance for the coprecipitation synthesis of Fe-oxide NPs using an inorganic base, $\mathrm{NH}_{3}$, and an organic base, isopropanolamine (MIPA).

\section{EXPERIMENTAL APPARATUS}

The developed AC susceptometer comprises a magnetizing (primary) and two sensing pick-up (secondary) coils oppositely wound and electrically connected in series positioned in the middle of the primary coils (see Fig. 1). Due to the identical characteristics of the secondary coils (6000 turns, $15 \mathrm{~mm}$ of internal diameter, $28 \mathrm{~mm}$ of external diameter and $25 \mathrm{~mm}$ height), the electromotive force $(\varepsilon)$ induced across the pick-up coils is ideally null if no magnetic sample is placed inside. In practice, it is impossible to wind identical coils resulting in a slight offset voltage across the secondary coils in the absence of a sample. On the other hand, inserting a magnetic sample into one of the sensing coils results in the generation of a non-zero signal according to the Faraday law,

$$
\varepsilon=-\frac{d \Phi}{d t}
$$

where $d \Phi / d t$ represents the change of magnetic flux across the sensing coils. A fundamental constraint of the coilassembly is that the magnetizing field $(H)$ should be as homogeneous as possible over the entire sample volume. For this reason, the primary coils in our system are Helmholtz coils (825 turns, $110 \mathrm{~mm}$ of diameter and 30 $\mathrm{mm}$ height) that assure a homogeneous applied magnetic field. These magnetizing coils are fed by an AC sinusoidal electrical current $(I)$ supplied by a Kepco source (KEPCO BOP 50-2M) controlled by a signal function generator (Model DS360 Standford Research System) which enables to set the amplitude $\left(V_{p p}\right)$, frequency $(f)$, and shape of $H$ (see Fig. 2).
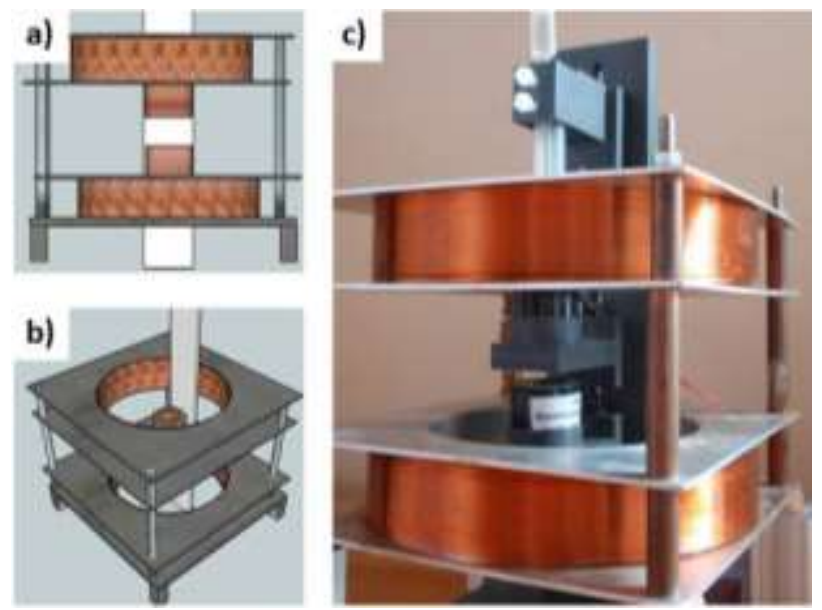

FIG. 1. (Color online) Schematic representation of a) lateral and b) 3D views of the Helmholtz and pick-up sensing coils. c) Picture of the actual set-up (except of electronic components, computer and pneumatic system). A detailed description of the dimensions and characteristics of the coils is given in the text.

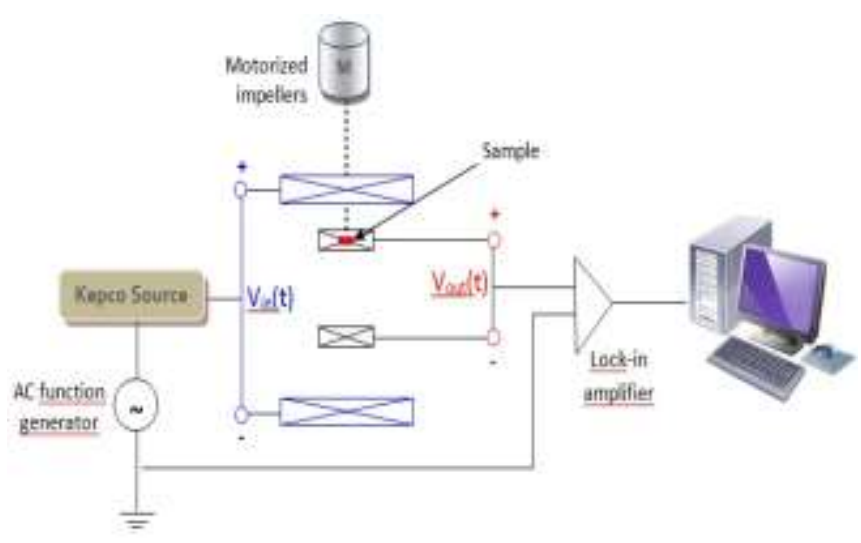

FIG. 2. (Color online) Scheme of the AC susceptometer apparatus. A detailed description of the device is presented in the text.

Figure 3 shows the behavior of the magnetic field amplitude $\left(H_{0}\right)$ produced by the Helmholtz coils as a function of its frequency for peak-to-peak amplitudes $\left(V_{p p}\right)$ between 2 and $10 \mathrm{~V}$. The signal across the pick-up coil $\left[V_{\text {out }}(t)\right]$ is then read by a digital lock-in amplifier (SR850 model Standford Research System), which acts as a phasesensitive voltmeter, and is transmitted to a computer via a GPIB interface. The reference signal is the AC sinusoidal voltage supplied by the signal generator. 


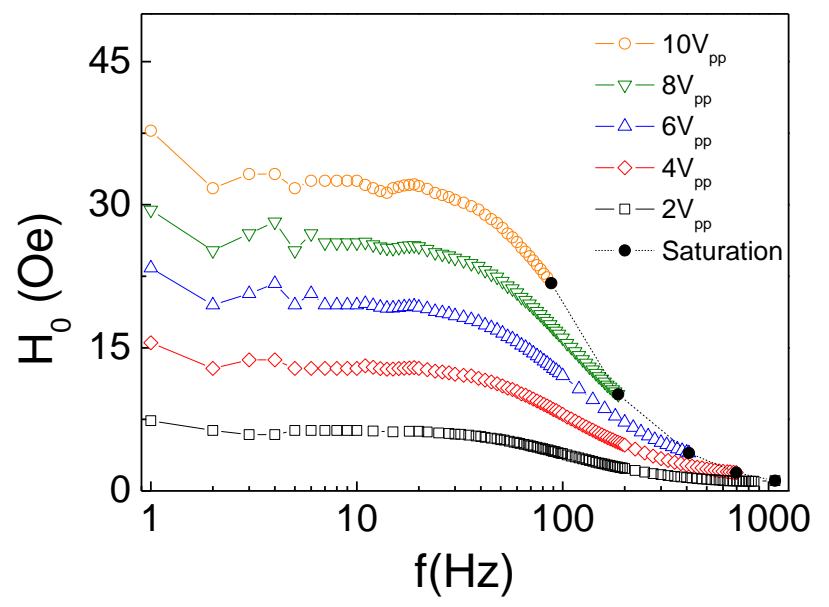

FIG. 3. (Color online) Behavior of the magnetizing field $\left(H_{0}\right)$ created by the primary (Helmholtz) coils as a function of its frequency $(f)$ for different peak-to-peak voltages $\left(V_{\mathrm{pp}}\right)$ of the signal generator.

The automatization of the AC susceptometer and the acquisition of the data are performed through a homemade LABVIEW program (Fig. 4). It is organized in three main parts: i) lock-in control parameters; ii) function signal generator control parameters; iii) measurement settings and display. In the first part, the required settings include: time constant, sensitivity, dynamic reserve, filter slope, autophase, autogain, autoreserve, auto offset and signals to be displayed in the lock-in front panel. In the second part, we can define the amplitude and frequency of the magnetizing field. Finally, the third has four displays related with the time dependence of the in-phase $(X)$ and out-of-phase $\quad(Y) \quad$ signals, $\quad R=\sqrt{X^{2}+Y^{2}}$ and $\theta=\tan ^{-1}(Y / X)$. The phase is the difference between the reference and the pick-up coils signals. It also includes measurement settings like acquisition and target time.

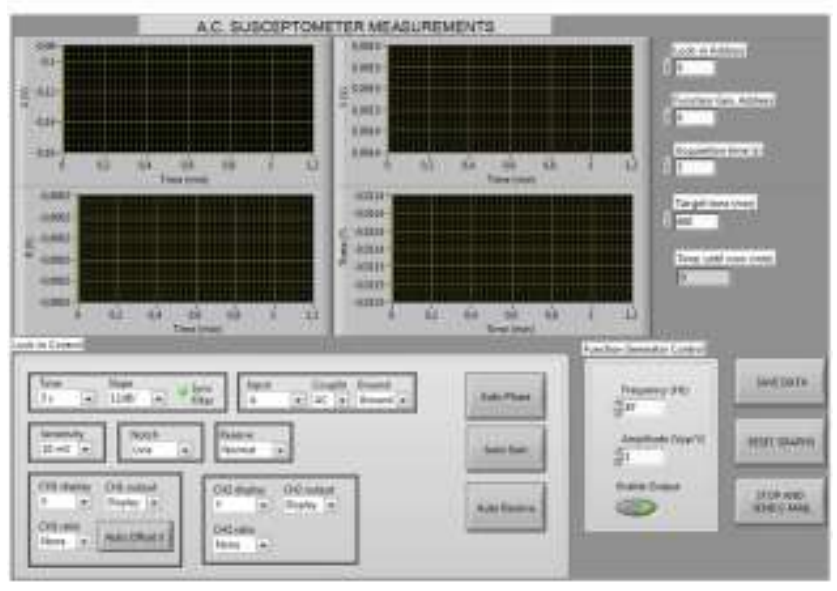

FIG. 4. (Color online) LABVIEW program for the automatization of the AC susceptometer unit.

\section{MAGNETIC SIGNAL DETECTED BY THE SENSING PICK-UP COILS}

The voltage signal induced on each pick-up coil is related with the magnetic flux $\Phi$, created by the AC magnetizing field produced by the primary coils, through Eq. (1). In the absence of a magnetic sample, the detected signal is ideally zero, as explained in section II. Imperfections in the construction of the secondary coil result in a small unbalanced signal that must be electronically compensated at the beginning of each experience with the auto-offset button. However, when a magnetic sample is placed inside one of the pickup coils and fits exactly its area, the differential generated signal $\left[V_{\text {out }}(t)\right]$ is described by:

$$
\begin{aligned}
V_{\text {out }}(t) & =-N S \frac{\partial\left(B_{2}-B_{1}\right)}{\partial t} \equiv N S \mu_{0} \frac{\partial M}{\partial t} \equiv \\
& \equiv N S \mu_{0} \kappa \frac{\partial H}{\partial t}
\end{aligned}
$$

where $N, S, B, \mu_{0}, M$ and $\kappa$ are the number of turns, crosssection area, magnetic field intensity, magnetic permeability of vacuum, magnetization and dimensionless magnetic susceptibility, respectively. Therefore, the differential signal, $V_{\text {out }}(t)$, detected by the lock-in amplifier has the form:

$$
V_{\text {out }}(t)=N S \mu_{0} \kappa H_{0} \omega \cos \omega t
$$

where $H$ has a sinusoidal shape with amplitude $H_{0}$ and angular frequency $\omega=2 \pi f$. In these expressions we are assuming that just one frequency component created by the magnetic field is induced in the secondary coils. The $V_{\text {out }}(t)$ signal is then compared within the lock-in amplifier with the sinusoidal voltage supplied by the AC signal function generator responsible for the creation of the sinusoidal magnetizing field. The magnitude and phase of the lock-in output voltage are calculated from the secondary $V_{\text {out }}(t)$ signal when the primary coils signal is at $0^{\circ}$ (in-phase; $X$ ) and $90^{\circ}$ (out-of-phase; $Y$ ), according to:

$$
\begin{aligned}
& X=\frac{G}{\sqrt{2}} N S \mu_{0} \kappa H_{0} \omega \cos \theta \\
& Y=\frac{G}{\sqrt{2}} N S \mu_{0} \kappa H_{0} \omega \sin \theta
\end{aligned}
$$

where $G$ represents the gain of the lock-in amplifier and $\theta$ takes into account phase shifts between the pick-up signal and the exciting (primary) signal.

\section{EXAMPLES}

A compromise must exist between the sensitivity of the lock-in and the magnetizing field parameters to increase the signal-to-noise ratio. Other lock-in parameters (e.g. time constant) and functions (e.g. dynamic reserve) can also be used to improve the signal-to-noise ratio. Attending to the materials used in this work and the sensitivity 
requirements, the measurements were made at $f=87 \mathrm{~Hz}$ and $V_{p p}=2 \mathrm{~V}$ (equivalent to $H_{o}=4.25 \mathrm{Oe}$, i.e. $338 \mathrm{~A} \cdot \mathrm{m}^{-1}$, see above).

The operation procedure is the following: $i$ ) the values of the $X, Y, R$ and $\theta$ channels are zeroed through autooffset and auto-phase buttons and, immediately after, the data started to be saved; ii) after $\sim 90 \mathrm{~s}$ of recording null reference signals, the sample is gently placed inside the upper secondary coil and we clearly distinguish that the recorded magnitudes vary; iii) few seconds after their stabilization, the sample is softly retrieved and we note that the displayed values of $X, Y, R$ and $\theta$ return to zero.

To further illustrate this procedure and verify the performance and resolution of our home-made setup, we tested both bulk and nanometric samples: (A) $\mathrm{Cu}$ and $\mathrm{Fe}$ bulk materials with approximately the same mass; (B) powder and ferrofluid of magnetic iron oxide and cobalt(II) ferrite NPs.

\section{A. Bulk magnetic materials}

Our setup measures detectable signals when the sample is "inside" and null when it is "outside" the sensing coil. Furthermore, it allows us to differentiate easily between diamagnetic $(M=\kappa \cdot H$, with $\kappa<0)$ and paramagnetic $(\kappa>$ 0 ) materials due to the sign of the $X$ signal (negative or positive, respectively) [see Eq. (4)].

We measured commercial samples purchased from Alfa Aesar: diamagnetic $\mathrm{Cu}$ wire sample (purity of $99 \%$, length $=120 \mathrm{~mm}$ and $\mathrm{m}=3.42 \mathrm{~g}$ ) and also a ferromagnetic Fe sample (purity of $98 \%, \mathrm{~m}=3.39$ g). Fig. 5 illustrates the $X$ and $\theta$ measured signals of the $\mathrm{Cu}$ and $\mathrm{Fe}$ powder bulk materials. As expected, the measured $X$-signal for $\mathrm{Cu}$ is negative due to the diamagnetism (see Fig. 5a). Furthermore, according to Eq. (4) we obtain an experimental and dimensionless magnetic susceptibility $\kappa(\mathrm{Cu})=-11.5 \times 10^{-7}$ which is in good agreement with the theoretical $\kappa(\mathrm{Cu})=-7.7 \times 10^{-7}$ (Ref. 31) revealing the trustworthy of our home-made set-up. Note that the eddy currents could not be discarded and contribute to an enhancement of the diamagnetic effect. On the other hand, the magnetization does not respond linearly to the applied magnetic field for ferromagnetic materials ( $\kappa=d M / d H$ ) and therefore, both in-phase and out-ofphase detectable signals are expected for Fe (see $X$ - and $\theta$ signals on Figs. 5c and 5d, respectively).

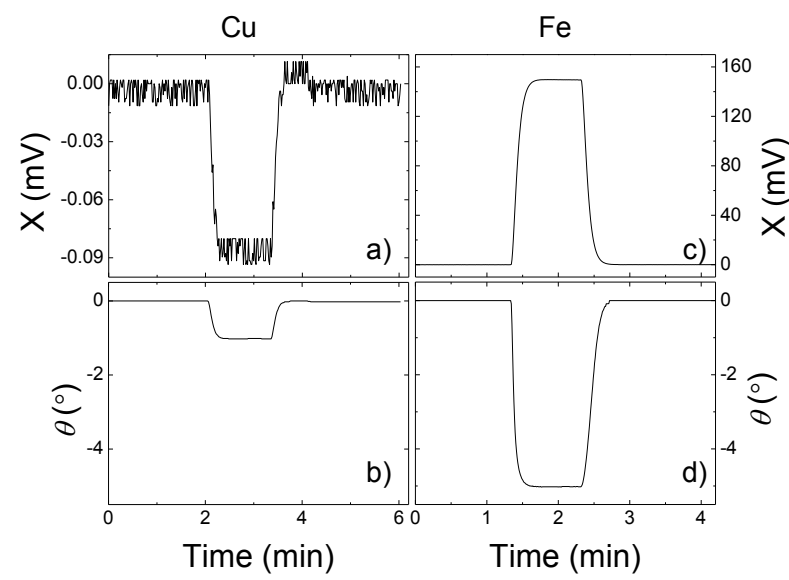

FIG. 5. Measured in-phase $(X)$ and phase difference $(\theta)$ signals of bulk $\mathrm{Cu}$ [a) and b), respectively] and $\mathrm{Fe}[\mathrm{b}$ ) and d), respectively] commercial materials with approximately the same mass $(\mathrm{m} \sim 3.4 \mathrm{~g})$ (see text).

\section{B. Fe-oxide magnetic nanoparticles}

Bearing in mind the reliability of our home-made setup to measure bulk magnetic materials, the accuracy of our AC susceptometer was tested with two magnetic samples of iron oxide and cobalt(II) ferrite NPs. Firstly, we measured commercial ferrimagnetic $\mathrm{Fe}_{2} \mathrm{O}_{3}$ NPs purchased from Alfa Aesar (constituted by $98 \%$ of $\gamma$-phase and thus we considered it as being maghemite). Secondly, an aqueous ferrofluid of Co-ferrite $\mathrm{NPs}, \mathrm{CoFe}_{2} \mathrm{O}_{4}$ prepared using MIPA $(3 \mathrm{M})$ as base was tested (see details of synthesis conditions on Ref. 23). Both samples exhibit dissimilar physical state $(63 \mathrm{mg}$ of powder and $2 \mathrm{ml}$ of magnetic ferrofluid, respectively) and, the mean NP dimensions are also different (between 20-40 $\mathrm{nm}$ for maghemite according to Alfa Aesar specifications and $\sim 4$ $\mathrm{nm}$ for $\mathrm{CoFe}_{2} \mathrm{O}_{4}$, respectively). Consequently, the NPs magnetism is also different: ferrimagnetic and superparamagnetic, respectively. We had previously characterized these samples by means of SQUID magnetometry and concluded that maghemite NPs exhibited a room temperature saturation magnetization of $M_{s}=68 \mathrm{Am}^{2} \mathrm{~kg}^{-1}$ and $H_{c}=0.01 \mathrm{~T}$. On the other hand, we have reported ${ }^{23,25}$ that due to their tiny dimensions $\mathrm{CoFe}_{2} \mathrm{O}_{4} \mathrm{NPs}$ displayed superparamagnetic behavior $\left(H_{c} \sim\right.$ $0 \mathrm{~T})$ and $M_{s}=50 \mathrm{Am}^{2} \mathrm{~kg}^{-1}$ at $300 \mathrm{~K}$.

Figure 6 shows the $X$ and $\theta$ measured signals of $\gamma$ $\mathrm{Fe}_{2} \mathrm{O}_{3}$ and $\mathrm{CoFe}_{2} \mathrm{O}_{4}$ NPs. Although we employed the same experimental conditions $\left(V_{\mathrm{pp}}=2 \mathrm{~V}\right.$ and $\left.f=87 \mathrm{~Hz}\right)$, the measured in-phase signals of magnetic NPs are orders of magnitude below that obtained for bulk $\mathrm{Fe}$, due to the reduced material quantity that was measured. This point is specially noticed on the $X$-signal to noise ratio of the ferrofluid sample shown on Fig. 6c. However, as in the case study of the ferromagnetic Fe sample, positive inphase signals were detected for both ferrimagnetic [Fig. 6a)] and superparamagnetic [Fig. 6c)] NPs. Finally, we detected an out-of-phase signal for the ferrimagnetic NPs [Fig. 6b)], whereas $\theta$ remained almost null (or at least under the detection limit of our set-up) for superparamagnetic NPs [see Fig. 6d)]. Consequently, the analysis of the $\theta$-signal provides interesting information 
and allows inferring the superparamagnetic character of the measured NPs (negligible $\theta$-signal when there is no phase shift between the measured and the exciting signals). ${ }^{32}$

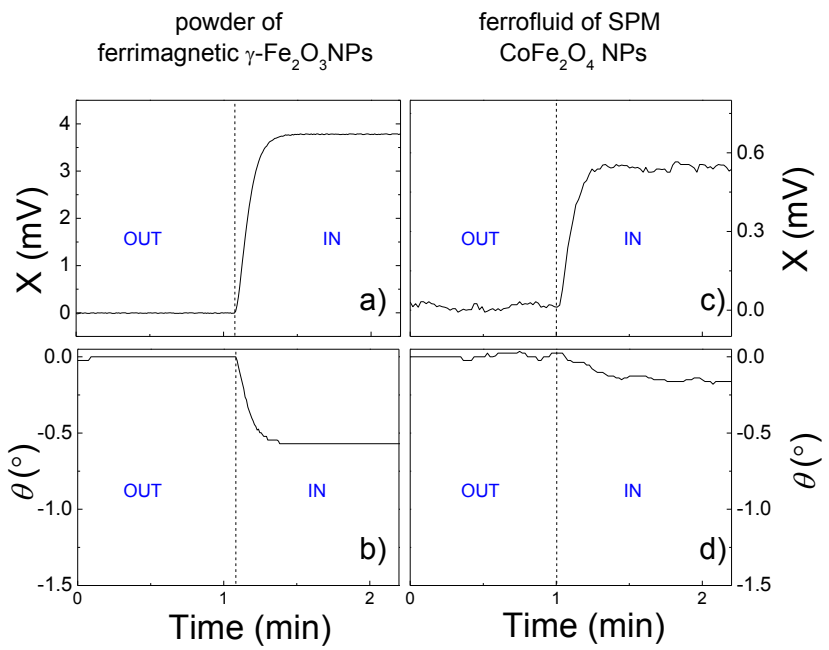

FIG. 6. (color online) Measured in-phase $(X)$ and phase difference $(\theta)$ signals of powder maghemite $\left(\gamma-\mathrm{Fe}_{2} \mathrm{O}_{3}\right)$ NPs purchased from Alfa Aesar [a) and b), respectively] and aqueous ferrofluid $(\mathrm{V}=2 \mathrm{ml})$ of $\mathrm{CoFe}_{2} \mathrm{O}_{4}$ NPs [c) and d), respectively]. The dashed lines indicate when the samples were placed "inside" the sensing coil (see text).

\section{MONITORING THE SYNTHESIS OF MAGNETIC IRON OXIDE NANOPARTICLES}

The synthesis of magnetic Fe-oxide NPs by coprecipitation reaction may be drastically altered through the variation on the reaction parameters (type of Fe salts; ratio of $\mathrm{Fe}(\mathrm{II}) / \mathrm{Fe}(\mathrm{III})$, reaction temperature; type and concentration of the base, etc.). In that sense, our set-up offers the unique opportunity of studying the influence of the preparation conditions on the physicochemical properties of the NPs in situ and in real time. Additionally, the in-phase and out-of-phase signals provide important information of the NPs' magnetic response (superparamagnetic or ferrimagnetic) reducing the number of subsequent characterizations.

\section{A. Importance of mixing}

It has been previously studied the importance of mixing vigorously the iron cations and alkaline solution during coprecipitation reactions to achieve a narrow particle size distribution. ${ }^{30,32}$ For that purpose, V. Ström and co-authors developed an external mixer to impinge two jets (metal salt precursors and base) and afterwards, transferred the reaction mixture to a sample vial that was placed inside the AC susceptometer. On the contrary, our set-up monitors the coprecipitation reaction from the exact moment that the base starts to be vigorously stirred, meanwhile the discharge of the $\mathrm{Fe}$ solution and, until the reaction is finished. For that purpose, we had to design and implement a hydrofoil impeller to promote a fast homogenization of the reaction mixture and produce a confined axial flow and low shear (see schematic picture on Fig. 7). This impeller has four blades with rounded leading edges at an angle of $45^{\circ}$ from the horizontal. The aqueous solution containing the iron(II) and iron(III) salts is discharged by means of a Teflon thin tube connected to a pneumatic system (syringe connected to a pneumatic pump). The tip of this Teflon tube is placed inside the alkaline solution and near the blades to avoid initial coalescence, agglomeration and flocculation. The impeller is connected to an AC motor and twirls inside the sample vial where the chemical reaction takes place. Within this impeller we uniformly mix the reagents and base in order to achieve narrow particle size distributions.

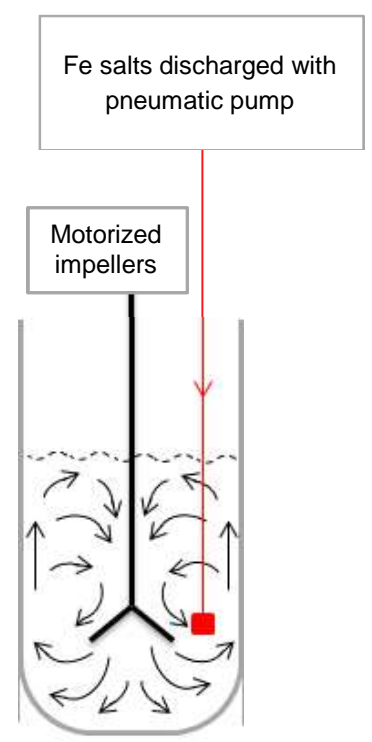

FIG. 7. (Color online) Scheme of the motorized impeller with four-blades placed at an angle of $45^{\circ}$ from the horizontal. The arrows indicate the schematic flow pattern created with the hydrofoil impeller. The discharge of the aqueous solution containing the Fe(II) and Fe(III) salts, that is controlled with a pneumatic pump, is done inside the liquid and near the blade where the local energy is large.

\section{B. Chemicals and reagents}

Iron(II) chloride tetrahydrate and iron(III) chloride hexahydrate (analytical grade) were supplied by Merk and Riedel-de Haën, respectively. Hydrochloric acid (37\%, analytical grade) was purchased from Panreac; ammonia solution (28\%) from Analar nanopure and 1-amino-2propanol (MIPA, 93\%) from Aldrich. Ultrapure water (Millipore with specific resistivity of $18 \mathrm{~m} \Omega \cdot \mathrm{cm}$ ) was used throughout the experiments. We employed all reagents without any further purification.

Two samples of magnetic NPs were prepared by coprecipitation using MIPA and $\mathrm{NH}_{3}$ as alkaline agents. For the synthesis of both iron oxide samples with different types of bases, the $\mathrm{Fe}(\mathrm{II}): \mathrm{Fe}(\mathrm{III})$ ratio was $1: 2$ corresponding to magnetite, $\mathrm{Fe}_{3} \mathrm{O}_{4}$. Firstly, $10 \mathrm{mmol}$ of $\mathrm{FeCl}_{2} \cdot 4 \mathrm{H}_{2} \mathrm{O}$ and $20 \mathrm{mmol}$ of $\mathrm{FeCl}_{3} \cdot 6 \mathrm{H}_{2} \mathrm{O}$ were dissolved independently in $12.5 \mathrm{ml}$ of deoxygenated aqueous solution of $\mathrm{HCl}$. Afterwards, both Fe chloride solutions were mixed leading to a final total iron concentration of 
1.2 M. This solution was protected from ambient atmosphere conditions by an argon gas flow. Secondly, deoxygenated $3.3 \mathrm{M}$ of MIPA solution or $2.5 \mathrm{M}$ of ammonia were prepared.

\section{In situ synthesis of iron oxide nanoparticles}

The operation procedure differs a little from that exposed on section IV: $i$ ) the $X, Y, R$ and $\theta$ channels are only zeroed after the test vial (containing the base solution), the Teflon thin tube (connected to the pneumatic system) and, the motorized impeller were placed inside the sensing coil; ii) Data started to be saved at the same time the chemical reaction started (i.e. the discharge of the aqueous solution containing the iron(II) and iron(III) chloride salts) and until the software was interrupted by the operator. This procedure allows us to follow in situ the total reaction from the first $\mu s$ of nucleation stage. Initially, $2 \mathrm{ml}$ of alkaline solution (2.5 $\mathrm{M} \mathrm{NH}_{3}$ or $3.3 \mathrm{M} \mathrm{MIPA}$ ) was stirred inside the sample vial. Next, the discharge of iron salts solution $(0.2 \mathrm{ml})$ was controlled by a pneumatic pump. The discharge was performed instantaneously ( 1 s) to the sample vial and the reaction was kept at room temperature. The studied volume was always the same because this is the maximum one that does not overflow the pickup coil dimensions, fitting exactly into their inner area.

Figure 8 displays the real time coprecipitation reactions during the synthesis of Fe-oxide NPs using $2.5 \mathrm{M} \mathrm{NH}_{3}$ and 3.3 M MIPA as bases. The evolution of the $X$ and $\theta$ with time reveals that the reactions are completely different for both chemical bases. The chemical reaction with ammonia is very fast in the first minute and finishes after $\sim 5 \mathrm{~min}$ (stable $X$ and $\theta$ signals). On the contrary, the in-phase signal for the reaction mixture using MIPA base increases gradually and does not stabilize even after 12 min (see Fig. 8a). In fact, the reaction for 3.5 M MIPA requires $100 \mathrm{~min}$ to be completed (not shown).

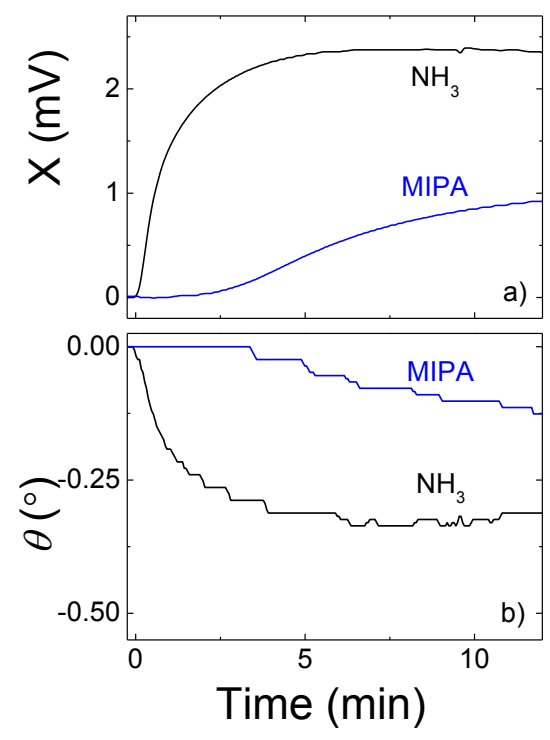

FIG. 8. (color online) Comparison of $X$ (a) and $\theta$ (b) signals of in situ coprecipitation reactions for the synthesis of iron oxide NPs using $\mathrm{NH}_{3}$ and MIPA as bases, monitored with our home-made AC susceptometer. Total iron cations concentration was $1.2 \mathrm{M}$. Base concentrations: $2.5 \mathrm{M}$ $\mathrm{NH}_{3}$ and 3.3 M MIPA.

On the other hand, the evolution of the $\theta(t)$ signals is rather the same as that of $X(t)$ (see Fig. $8 \mathrm{~b}$ ): very fast for $\mathrm{NH}_{3}$ and rather slow for MIPA. The phase difference reaches a stable value of $\theta \sim-0.33^{\circ}$ after $5 \mathrm{~min}$ for ammonia and $-0.15^{\circ}$ after $1 \mathrm{~h}$ for MIPA (not shown). The out-of-phase evolution is an indicative of a developing coercivity and thus, particle growth. ${ }^{30}$ Therefore, we can predict minute coercivities and reduced mean particle dimensions for both samples of Fe-oxide NPs. ${ }^{32}$ In fact, the histograms obtained from the analysis of the diameter of more than $>1000$ NPs on TEM images (not shown) reveal narrow distributions centered at $\mu(\sigma)=3.5(0.4) \mathrm{nm}$ for 2.5 $\mathrm{M}$ ammonia-based sample and 2.7(0.2) nm for $3.5 \mathrm{M}$ MIPA-based sample. At the same time, we have demonstrated that the standard time of $2 \mathrm{~h}$ (typically employed on coprecipitation reactions) could be drastically reduced depending on the synthetic conditions.

\section{SUMMARY}

In summary, we have designed and built a relatively simple AC susceptometer to study in situ coprecipitation reactions. For that purpose, we had to implement a hydrofoil impeller with $45^{\circ}$-angle blades to promote the fast homogenization of the reaction mixture. Our homemade set-up opens the possibility of exploring easily and in real time how the dynamic of the chemical reaction is influenced by the type of metal cations, base and their relative concentrations. Therefore, future work could be done to model the magnetic response of the NPs rapidly avoiding long-term physicochemical characterizations after synthesis. Our work opens the possibility of scalingup our AC susceptometer to monitor in situ the synthesis of magnetic NPs on large scale. Moreover, the set-up could be slightly modified in order to be implemented during the full course of chemical reactions for the synthesis of other types of magnetic NPs.

\section{ACKNOWLEDGEMENTS}

The authors thank the mechanical and electrical work developed at the workshop of the Faculty of Sciences of the University of Porto. This work was supported by FCT through project ref. PTDC/QUI-QUI/105304/2008 and through grants no. PEst-C/EQB/LA0006/2013 and FCOMP-01-0124-FEDER-037285. The authors acknowledge Operations NORTE-070124-FEDER000070-MULTIFUNCTIONAL NANOMATERIALS and NORTE-07-0124-FEDER-000067 - NANOCHEMISTRY funded by FEDER and CCDRN. MPFG and JMT thank FCT for their grants (SFRH/BPD/87430/2012 and SFRH/BPD/72329/2010, respectively); MRO and JMM thank FCUP for being awarded with extracurricular stages at IFIMUP-IN (PEEC-2012 and PEEC-2013, respectively); PM also acknowledges FCT for being 
granted through PTDC/QUI-QUI/105304/2008 and PTDC/FIS-NAN/0533/2012.

\section{REFERENCES}

${ }^{1}$ J.L. Dormann, D. Fiorani, E. Tronc, Magnetic Relaxation in fine-particle systems Vol. 98 (John Wiley \& Sons, 1997).

${ }^{2}$ R.H. Kodama, J. Magn. Magn. Mater. 200, 359 (1999).

${ }^{3}$ Y.-W. Jun, Y.-M. Huh, J.-S. Choi, J.-H. Lee, H.-T. Song, S. Kim, S. Yoon, K.-S. Kim, J.-S. Shin, J.-S. Suh, J. Cheon, J. Am. Chem. Soc. 127, $5732(2005)$.

${ }^{4}$ A.K. Gupta, M. Gupta, Biomaterials 26(18), 3995 (2005).

${ }^{5}$ D.L. Huber, Small 1(5), 482 (2005).

${ }^{6}$ C.T. Yavuz, J.T. Mayo, W.Y. William, A. Prakash, J.C. Falkner, S. Yean, L. Cong, H.J. Shipley, A. Kan, M. Tomson, D. Natelson, V.L. Colvin, Science 314, 964 (2006).

${ }^{7}$ M.P. Fernandez, D.S. Schmool, A.S. Silva, M. Sevilla, A.B. Fuertes, P. Gorria, J.A. Blanco, J. Non. Cryst. Solids 354(47), 5219 (2008).

${ }^{8}$ S.F. Chin, K. Swaminathan Iyer, C. L. Raston, M. Saunders, Adv. Funct. Mater. 18, 922 (2008).

${ }^{9}$ L.-M. Lacroix, J.Carrey, M.Respaud, Rev. Sci. Instrum. 79, 093909 (2008).

${ }^{10}$ M.P. Fernandez, D.S. Schmool, A.S. Silva, M. Sevilla, A.B. Fuertes, P. Gorria, J.A. Blanco, J. Magn. Magn. Mater. 322(9), 1300 (2010).

${ }^{11}$ M.P. Fernandez-Garcia, P. Gorria, J.A. Blanco, A.B. Fuertes, M. Sevilla, R. Boada, J. Chaboy, D.S. Schmool, J.M. Greneche, Phys. Rev. B 81(9), 094418 (2010).

${ }^{12}$ J. Santoyo Salazar, L. Perez, O. Abril, L.T. Phuoc, D. Ihiawakrim, M. Vazquez, J.-M. Greneche, S. Begin-Colin, G. Pourroy, Chem. Mater. 23, 1379 (2011).

${ }^{13}$ M.P. Fernandez-Garcia, P. Gorria, M. Sevilla, M.P. Proenca, R. Boada, J. Chaboy, A.B. Fuertes, J.A. Blanco, J. Phys. Chem C 115(13), 5294 (2011).

${ }^{14}$ L. Harivardhan Reddy, J.L. Arias, J. Nicolas, P. Couvreur, Chem. Rev. 112, 5818 (2012).

${ }^{15}$ P. Tartaj, M.P. Morales, T. Gonzalez-Carreño, S. VeintemillasVerdaguer, C.J. Serna, Adv. Mater. 23, 5243 (2011).

${ }^{16}$ M. Fang, V. Ström, R. T. Olsson, L. Beova, K.V. Rao, Nanotechnology 23, 145601 (2012).

${ }^{17}$ P. de la Presa, Y. Luengo, M. Multigner, R. Costo, M.P. Morales, G. Rivero, A. Hernando, J. Phys. Chem. C 116(48) 25602 (2012).

${ }^{18}$ D. Ling, T. Hyeon, Small 9(9), 1450 (2013).

${ }^{19}$ A. Riedinger, P. Guardia, A. Curcio, M. A. Garcia, R. Cingolani, L. Manna, T. Pellegrino, Nano Lett. 13, 2399 (2013).

${ }^{20}$ Y.V. Kolenko, M. Bañobre-López, C. Rodríguez-Abreu, E. CarbóArgibay, A. Sailsman, Y. Piñeiro-Redondo, M. Fátima Cerqueira, D.Y. Petrovykh, K. Kovnir, O.I. Lebedev, J. Rivas, J. Phys. Chem. C 118, 8691 (2014).

${ }^{21}$ S. Dutz, R. Hergt, Nanotechnology 25, 452001 (2014).

${ }^{22}$ J. Zeng, L. Jing, Y. Hou, M. Jia, R. Qiao, Q. Jia, C. Liu, F. Fang, H. Lei, M. Gao, Adv. Mater. 26, 2694 (2014).

${ }^{23}$ C. Pereira, A.M. Pereira, C. Fernandes, M. Rocha, R. Mendes, M.P. Fernández-García, A. Guedes, P.B. Tavares, J-.M-. Grenèche, J.P. Araujo, C. Freire, Chem. Mater. 24, 1496 (2012).

${ }^{24}$ J.R. Eltzholtz, B.B. Iversen, Rev. Sci. Instrum. 82, 084102 (2011).

${ }^{25}$ C. Fernandes, C. Pereira, M.P. Fernández-García, A.M. Pereira, A. Guedes, R. Fernández-Pacheco, A. Ibarra, M.R. Ibarra, J.P. Araújo, C.

Freire, J. Mater. Chem. 2, 5818 (2014).

${ }^{26}$ J.J. Ibarra-Sánchez, R. Fuentes-Ramírez, A.G. Roca, M.P. Morales, L.I. Cabrera-Lara, Ind. Eng. Chem. Res. 52, 17841 (2013).

${ }^{27}$ G. Salazar-Alvarez, M. Muhammed, A.A. Zagorodni, Chem. Eng. Sci. 61, 4625 (2006).

${ }^{28}$ J. Park, J. Joo, S. G. Kwon, Y. Jang, T. Hyeon, Angew. Chem. Int. Ed. 46, 4630 (2007).

${ }^{29}$ S. Laurent, D. Forge, M. Port, A. Roch, C. Robic, L.V. Elst, R.N. Muller, Chem. Rev. 108, 2064 (2008).

${ }^{30}$ V. Ström, R.T. Olsson, K.V. Rao, J. Mater. Chem. 20, 4168 (2010).

${ }^{31}$ D.R.Lide ed., CRC Handbook of Chemistry and Physics (CRC Press, Boca Raton, FL, 2005).

${ }^{32}$ M. Fang, V. Ström, R.T. Olsson, L. Belova, K.V. Rao, Appl. Phys. Lett. 99, 222501 (2011). 